EPJ Web of Conferences 41, 02016 (2013)

DOI: $10.1051 /$ epjconf/20134102016

(C) Owned by the authors, published by EDP Sciences, 2013

\title{
Low-Energy Peak Structure in Strong-Field Ionization by Mid-Infrared Laser Pulses
}

\author{
C. Lemell ${ }^{1}$, K. I. Dimitriou ${ }^{2,3}$, D. G. Arbó ${ }^{4}$, X.-M. Tong ${ }^{5}$, D. Kartashov ${ }^{6}$, J. Burgdörfer ${ }^{1}$, S. Gräfe ${ }^{1}$ \\ ${ }^{1}$ Institute for Theoretical Physics, Vienna University of Technology, Wiedner Haupstr. 8-10, A- \\ 1040 Vienna, Austria \\ ${ }^{2}$ Department of Physical Science and Applications, Hellenic Army Academy, Vari, Greece \\ ${ }^{3}$ National Hellenic Research Foundation, Inst. of Theoretical and Physical Chemistry, Athens, \\ Greece \\ ${ }^{4}$ Institute for Astronomy and Space Physics, IAFE (FCEN-UBA Conicet), Buenos Aires, Argentina \\ ${ }^{5}$ Institute of Materials Science, University of Tsukuba, Ibaraki 305-8573, Japan \\ ${ }^{6}$ Photonics Institute, Vienna University of Technology, Gusshausstr. 27, A-1040 Vienna, Austria
}

\begin{abstract}
Using a quasiclassical approach, we demonstrate that the formation of the low-energy structure in above-threshold ionization spectra by intense, midinfrared laser pulses originates from a two-dimensional focusing of the strong-field dynamics in the energy-angular-momentum plane. We show that the low-energy structure is very sensitive to the carrier-envelope phase of the laser field.
\end{abstract}

\section{Introduction}

Strong-field ionization is one of the fundamental processes in the interaction of intense laser fields with matter [1]. Based on the analytic approach of Keldysh, Faisal and Reiss [2-4], most of the phenomena occurring in intense laser fields can be described in frame of the strong-field approximation (SFA). The SFA assumes that the influence of the laser electric field on the electrons after ionization is dominant and that the atomic potential can be neglected. The SFA is expected to work well for Keldysh parameters $\gamma=\omega \sqrt{2 I_{P}} / E_{0}<<1$, with $E_{0}$ and $\omega$ being the field strength and frequency, and $I_{p}$ the ionization potential of the atom. This corresponds to the so-called tunneling regime, where Coulomb effects are expected to be of minor importance. Very recently, an "ionization surprise" in form of an unexpected spike-like low-energy structure (LES) in abovethreshold ionization (ATI) spectra was found [5-7]. The structure occurs for mid-infrared wavelengths with $\lambda>1600 \mathrm{~nm}$, at moderate intensities (around $10^{14} \mathrm{~W} / \mathrm{cm}^{2}$ ), i.e. for Keldyshparameters $\gamma<1$. Most importantly, the LES cannot be described within standard SFA theory, although SFA is expected to hold particularly well in this regime.

We show with the help of classical trajectory Monte Carlo (CTMC) calculations that the LES is related to a classical two-dimensional focusing in phase space giving rise to a strong correlation between the energy $E$ and the angular momentum $L$ of the LES electrons. Moreover, we find a pronounced carrier-envelope phase (CEP) dependence of the LES.

This is an Open Access article distributed under the terms of the Creative Commons Attribution License 2.0, which permits unrestricted use, distribution, and reproduction in any medium, provided the original work is properly cited. 


\section{Numerical results}

For our classical simulations an adaptive step-size Runge-Kutta propagator is employed. The initial conditions are given by the tunnelling expressions by Delone and Krainov [8]: The initial coordinate on the polarization axis is the tunnel exit $\left(x_{0}=y_{0}=0, z_{0}=I_{p} / E_{0}\right)$; the initial momentum distribution are Gaussian distributions, centred around $p_{\mathrm{II}}, p_{\perp}=0$.

As a laser pulse we choose a cosine like pulse shape (carrier-envelope phase $\varphi_{\mathrm{CEP}}=0$ ) with a $\sin ^{2}$ envelope for the electric field, a wavelength of $\lambda=2200 \mathrm{~nm}$, a peak intensity of $I=10^{14} \mathrm{~W} / \mathrm{cm}^{2}$, and a duration of eight cycles (full width at half maximum (FWHM) of intensity $\sim 20 \mathrm{fs}$ ). Fig. 1 displays the final distribution in the energy $(E)$-angular momentum $(L)$ plane of electrons emitted into a double cone oriented along the polarization axis $\left(0^{\circ}\right.$ and $\left.180^{\circ}\right)$ with an opening angle $\theta c= \pm 10^{\circ}$. An island with three distinct peaks at high angular momenta (around $L=16$ ) can be identified. Projection onto the energy axis shows that this high- $L-$ low- $E$ island can be identified as the source of the LES with energy of $E \approx 3 \mathrm{eV}$. This island is well separated from the "background" of low-energy, lowangular-momentum electrons with sharp, parabolic boundary. The positions $\left(E_{i}, L_{i}\right)$ of the peaks within the island are determined by the variation of the subsequent field maxima within the pulse envelope; for longer pulses, the peaks merge, as will be demonstrated below.

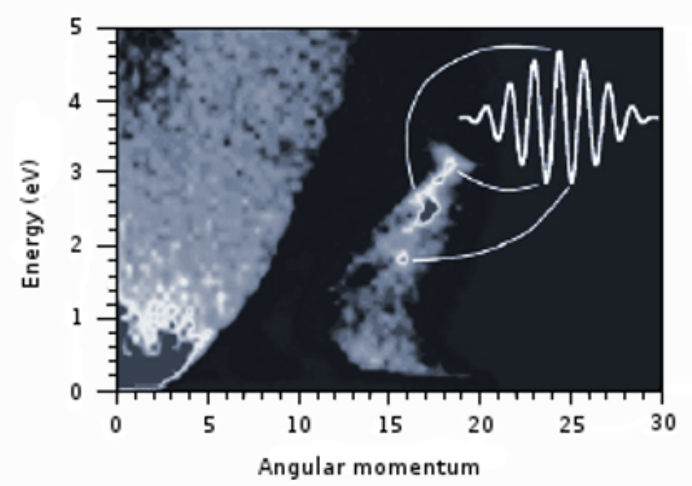

Figure 1 Electron distribution in the energy-angular momentum plane within an angular cone of $\theta c= \pm 10$. The low-energy - high-angular momentum island with the three peaks is the origin of the LES. Inset: electric field of the laser pulse $\left(2200 \mathrm{~nm}, I=10^{14} \mathrm{~W} / \mathrm{cm}^{2}, 20 \mathrm{fs}\right.$ FWHM). Adapted from Ref. [11].

The classical dynamics allows for an analysis of trajectories reaching the island: launched at the tunnel exit, they are driven back to the nucleus in close distance in the subsequent laser half-cycle. In the subsequent cycle, when the quiver motion is at an "inner" turning point in the vicinity of the ionic core, with momentum $p_{\mathrm{II}} \approx 0$, the Coulomb potential is the main force acting on the electron, and the trajectories are "soft-scattered" [9] being eventually locked on to Kepler hyperbolae of different orientations but similarly large angular momenta [10]

$$
L=\sqrt{\alpha^{2} p^{2}+2 \alpha}, \quad \text { thus for small } p: L \approx \sqrt{2 \alpha},
$$

with the quiver radius $\alpha=E_{0} / \omega^{2}$. Almost all electrons in the LES are accumulated around the same value of the angular momentum quantum number $L$, where $L$ scales with the quiver radius, and thus with the laser parameters. The scenario of strong-field quiver motion only locally perturbed near the "inner" turning points requires a large quiver amplitude $\alpha$ compared to the typical distance from the core, taken to be of the order of the tunnel exit $z_{0}$,

$$
\frac{\alpha}{z_{0}} \approx \frac{E_{0}^{2}}{I_{P}(2 \pi c)^{2}} \lambda^{2} \approx \frac{2}{\gamma^{2}}>1,
$$

and readily explains why a pronounced LES develops only for midinfrared pulses.

The low-energy structure occurs also in short-range potentials, if the range $d$ of the short-range potential extends to at least a distance on the order of the tunnel exit $\left(z_{0} \lesssim d<\alpha\right)$ such that the 
atomic field can impart a longitudinal momentum "kick" to accelerate and a transversal "kick" to deflect the outgoing trajectory at the inner turning point of the subsequent half-cycle.

From the trajectory analysis presented above, we expect different electron spectra in forward and backward direction. The trajectories forming the LES are "indirect" ones, trajectories which propagate asymptotically in the direction opposite to that of the tunnel exit. Thus, for a short cosine pulse $\left(\varphi_{\mathrm{CEP}}=0\right)$ the LES in both position and shape differs significantly from the sine-shaped pulse $\left(\varphi_{\mathrm{CEP}}=\pi / 2\right)$. Due to the energy shift of the LES per half cycle, these LES are observed at different energies [11]. This promises to give access to information on the CEP phase $\varphi_{\mathrm{CEP}}$. For pulses with longer wavelengths, the low-energy region features even richer structure, as multiple peaks are revealed which can be explained to higher-order soft-rescattering events.

\section{Conclusions}

The LES occurs for large ratios of quiver amplitude to distances of the tunnel exit. The strongfield quiver dynamics is locally perturbed by the ionic potential near the "inner turning point" during the half-cycles subsequent to the one causing ionization. The distribution of electrons forming the LES features an accumulation around the same high angular momentum value $L$, the latter being determined by the laser parameters. The low-E-high- $L$ island originates from a two-dimensional focusing in phase space, mapping the initial conditions, time $t$ (or laser phase $\phi=\omega t$ ), and the initial transverse momentum $p_{\perp}$ with which the trajectory is launched onto the final-state variables $E$ and $L$. This phase-space focusing can be directly visualized by the distortion (stretching and compression) of the initial phase space volume when mapped onto the $E-L$ plane, see Ref. [11].

The trajectory analysis of the LES opens up novel opportunities to monitor the carrier-envelope phase of ultrashort few-cycle midinfrared pulses. As trajectories asymptotically propagate in the direction opposite to that of the tunnel exit ("indirect trajectories"), different spectra in forward (defined by the direction of the maximum laser field) and backward directions can be expected.

Acknowledgements: S. G. acknowledges financial support by the Austrian Science Fund FWF, project number V193-N16.

1. W. Becker, F. Grasbon, R. Kopold, D. B. Milosevic, G. G. Paulus, and H. Walther, Adv. Mol. Opt. Phys. 48, 35 (2002).

2. L. V. Keldysh, J. Exptl. Theoret. Phys. (USSR) 47 (1964) 1945, English translation: Soviet Physics JETP 20 (1965) 1307.

3. F. H. M. Faisal, J. Phys. B: At. Mol. Phys. 6, L89 (1973).

4. H. B. van Linden van den Heuvell and H. G. Muller, in Multiphoton Processes, edited by S. J. Smith and P. L. Knight (Cambridge University Press, Cambridge UK, 1988).

5. W. Quan, Z. Lin, M. Wu, H. Kang, H. Liu, X. Liu, J. Chen, J. Liu, X. T. He, S. G. Chen, H. Xiong, L. Guo, H. Xu, Y. Fu, Y. Cheng', and Z. Z. Xu, Phys. Rev. Lett. 103, 093001 (2009).

6. C. I. Blaga, F. Catoire, P. Colosimo, G. G. Paulus, H. G. Muller, P. Agostini, and L. F. DiMauro, Nat. Phys. 5, 335 (2009).

7. F. Catoire, C. I. Blaga, E. Sistrunk, H. G. Muller, P. Agostini, and L. F. DiMauro, Laser Phys. 19, 1574 (2009).

8. N. B. Delone, V. P. Krainov, J. Opt. Soc. Am. B 8, 1207 (1991).

9. A. Kästner, U. Saalmann, and J. M. Rost, Phys. Rev. Lett. 108, 033201 (2012).

10. D. G. Arbó, S. Yoshida, E. Persson, K. I. Dimitriou, and J. Burgdörfer, Phys. Rev. Lett. 96, 143003 (2006).

11. C. Lemell, K. I. Dimitriou, X.-M. Tong, S. Nagele, D. V. Kartashov, J. Burgdörfer, and S. Gräfe, Phys. Rev. A 85, 011403(R) (2012). 